\title{
RAFTER: Ring Astrometric Field Telescope for Exo-planets and Relativity
}

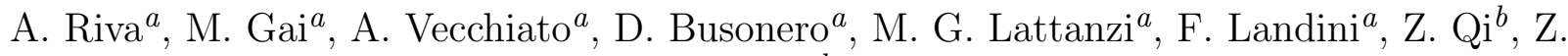 \\ Tang $^{b}$ \\ ${ }^{a}$ Istituto Nazionale di Astrofisica - Osservatorio Astrofisico di Torino, V. Osservatorio, 20, \\ I-10025 Pino Torinese (TO), Italy \\ ${ }^{b}$ Shanghai Astronomical Observatory, Chinese Academy of Sciences, 80 Nandan Road, \\ Shanghai 200030, China
}

\begin{abstract}
High precision astrometry aims at source position determination to a very small fraction of the diffraction image size, in high SNR regime. One of the key limitations to such goal is the optical response variation of the telescope over a sizeable FOV, required to ensure bright reference objects to any selected target. The issue translates into severe calibration constraints, and/or the need for complex telescope and focal plane metrology. We propose an innovative system approach derived from the established TMA telescope concept, extended to achieve high filling factor of an annular field of view around the optical axis of the telescope. The proposed design is a very compact, $1 \mathrm{~m}$ class telescope compatible with modern CCD and CMOS detectors (EFL = $15 \mathrm{~m}$ ). We describe the concept implementation guidelines and the optical performance of the current optical design. The diffraction limited FOV exceeds 1.25 square degrees, and the detector occupies the best 0.25 square degree with 66 devices.
\end{abstract}

Keywords: Astrometry, Fundamental Physics, Metrology

\section{INTRODUCTION}

Astrometric measurements at or below the micro-arcsec $(\mu a s)$ level appear to be compatible with even moderate values of telescope diameter and exposure time, for observation of fairly bright stars in a wide spectral band in the visible range (as recalled below). We take as reference science case the search for Earth-like exo-planets in the population of nearby (bright) stars with spectral type not too different from our Sun.

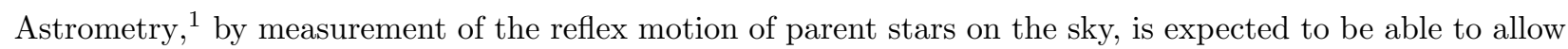
determination of individual planet's mass and orbital inclination, and ease the full solution of multiple systems. Competing techniques, as radial velocity and transits, are most sensitive to specific target geometries and/or astrophysical characteristics, so that most currently detected exo-planetary systems are quite different from our solar system.

This consideration motivates the proposition in recent years of projects like NEAT, $\stackrel{2]}{2}$ Theia, $\stackrel{3}{2}$ TOLIMAN 4 and others,,$\sqrt[5]{ }$ devoted to astrometric surveys e.g. of the limited sample of several ten nearest and brightest near-solar stars, in particular on already detected systems, in which $\sim 1$ mas measurement precision may allow accurate determination of Earth's analogues. We recall that for a $\sim 1 M_{\oplus}$ planet at $\sim 1 A U$ from a near-solar star, i.e. within its habitable zone $(\mathrm{HZ})$, the astrometric signal is $\sim 0.3$ pas. Some of the proposers are also engaged in the bilateral (China-Italy) research project ASTRA (Astrometric Science and Technology Roadmap for Astrophysics) ${ }^{[6}$ presented in another contribution to these Proceedings.

The limiting astrometric precision ${ }^{7} \sqrt{9}$ is associated to the instrument resolution and the photon budget. However, preserving a comparable measurement accuracy on the angular separation among stars is not so easy, since the realistic optical response of a system implies a Point Spread Function (PSF) variation over the field, due to aberrations, which affects the photo-centre determination. Also, the photons from different viewing directions, associated to different focal plane positions, use different parts of the optical system; the non-common parts of the optical paths are affected by further variations ("beam walk") due not only to the field dependence of

Further author information: E-mail: alberto.riva@inaf.it, Telephone: +39011 8101 963, www.oato.inaf.it 
aberrations, but also to the different characteristics of different areas of the optical surfaces, e.g. from figuring and micro-roughness. Actually, the knowledge on instrument response, by calibration and metrology, mitigates the problem, so that "only" residual effects are to be taken into account; unfortunately, they are still significant at the $\mu$ as level.

It should be noted that high precision astrometry implicitly requires a correct implementation of the measurement framework in the context of General Relativity (GR). Gravitational deflection of the photons ${ }^{10}$ by the space-time curvature induced by the Sun alone corresponds to several milli-arcsec (hereafter, mas) over most of the sky accessible by an observer in the vicinity of the Earth. In many cases, the effects of Solar system planets not far away from the observing direction may be significant at the (sub)- $\mu a$ s level.

The real challenges to accuracy stem from three main potential sources of systematic errors:

- Variable optical response of the telescope;

- Variable electro-optical response of detector;

- "Cosmic noise", i.e. intrinsic variability of astronomical objects;

The annular field telescope concept arises mainly as a possible approach toward answer to the first issue, although some considerations about the others will be proposed as well.

Optical response is of course one of the main design drivers, since usually science requirements are translated into specifications for image quality over a suitable field of view (FOV). The need for a minimum size of the instrument FOV is related to assuring the capability of observing several sources simultaneously, for reasons of efficiency and to provide references to the science target (calibration). Besides, the instrument response typically changes over a large FOV, both in terms of optical parameters and of detector characteristics, in turn affecting the calibration requirements.

Therefore, the requirement of good optical response, possibly uniform, over the whole field of view, is a system level challenge, from optical design, through manufacturing and testing, to integration and in-flight alignment. It remains a critical aspect throughout science operation, because instrument calibration must be maintained over the useful lifetime.

\subsection{Rationale for an annular field telescope}

In order to alleviate some of the above challenges, we investigate a novel instrument concept, in which a telescope is optimized on an annular region set at a given radius from the optical axis. In a nominal optical system characterized by circular symmetry, the optical response is also the same, apart rotation, over any concentric ring of the focal plane. We remark that the ring region corresponds to a small set of angular values, just replicated by symmetry. The annular field can provide simultaneous observation of any target pair with separation ranging from zero to its angular diameter. On the other hand, the annular field may not be convenient for some applications, e.g. to perform a uniform scan of a large area.

The optical design is based on the same optimization tools used to achieve conventional large field telescopes already presented in the literature, just by reformulation of the cost function, so that, upon convergence, the optical response can be expected to be as good, uniform and reliable as other configurations analyzed. We are investigating the feasibility of such design approach.

An application of the annular field telescope concept is presented in another contribution to these Proceedings, describing two configuration options for the AGP (Astrometric Gravitation Probe) ${ }^{11}$ mission concept.

An annular system with detectors covering the limited radial range between $\theta$ and $\theta+\delta \theta$ still provides a significant total area $2 \pi \theta \cdot \delta \theta$, but much less than that of the inscribed circle $\pi \theta^{2}$ when the ring is large with respect to its thickness, i.e. $\delta \theta<<\theta$. An example of annular field, with radius $\theta=1^{\circ}$, is shown in Fig. 1 . the ring width is $\delta \theta=3^{\prime} .75$. The same number of detectors used in the ring are also shown (in gray) in a compact distribution around the FOV center, for comparison with a conventional mosaic having comparable complexity. The total field of view is 0.258 square degrees, covered by 66 detectors, but in the conventional case (filled central 


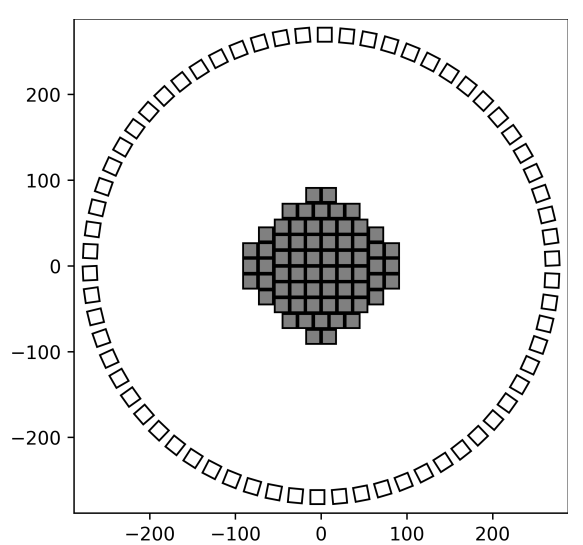

Figure 1. Ring focal plane with radius $1^{\circ}$, composed of 66 detectors, and the same devices (in gray) arranged around the optical axis. Plot axes are in $\mathrm{mm}$.

area, gray in figure) the maximum angular separation between simultaneously imaged sources is much smaller, i.e. $\sim 37$ arcmin against $2^{\circ}$.

It may be noted that any instrument aiming at filling one square degree with $\sim 50$ mas pixels is fated to end up with a pixel count of $\sim \pi(20 \times 3600)^{2} \simeq 1.6 \times 10^{10}$ pixels, i.e. $\sim 1,000$ individual $4 k \times 4 k$ chips. Availability of larger format devices would alleviate some of the system complexity, but not the total pixel count.

Even if negligible variation of the optical response over the focal plane were achieved, which is one of our key requirements, the sheer cost of such detection system is dramatically in favour of our alternative annular field approach.

The key point addressed in this paper is the feasibility and potential performance of the annular field approach, in particular with respect to optical design and system considerations. In Sec.2 we describe the design development guidelines and main results on optical performance; in Sec.3 we briefly review the highlights of the proposed concept, in particular concerning astrometric capabilities; finally, in Sec.4 we draw our conclusions outlining future investigations.

\section{CONCEPT DEVELOPMENT AND OPTICAL DESIGN}

The concepts investigated in the framework of NEAT ${ }^{2}$ and Thei ${ }^{3}$ (proposed respectively in response to ESA's M3 and M4 Calls) address the astrometric accuracy issues by selecting either a very simple telescope (one mirror), or a high performance system (three mirror anastigmat, TMA), providing a good optical response, uniform over a convenient FOV. Moreover, the instrument design was endowed with advanced metrology and calibration systems to monitor the astrometric response and allow correction of the still existing systematic errors in the data reduction.

In this paper, we build on similar concepts, exploiting the symmetry of an optical system derived from a TMA design, and introducing an optimization approach restricted to an annular area of the focal plane. A ring centered on the optical axis of a nominal on-axis system is basically considered as a single point with respect to optical quality, just replicated by circular symmetry over the selected circle. A narrow annular region can therefore be expected to feature a small radial variation in optical response, with very uniform characteristics in the azimuthal direction.

The optical design of large aperture, large field of view telescopes with three or four mirrors endowed with optical power, plus additional folding mirrors, has been consolidated in the past few decades, in particular with the concept of Three-Mirror Anastigmats (TMA) developed by D. Korsch 12 13 TMA telescopes have been adopted for important science experiments, e.g. the Euclid mission 14 and the James Webb Space Telescope (JWST) $\mathbb{1 5}^{15}\left[16\right.$ The GAME ${ }^{17}\left[18\right.$ and AGP ${ }^{19} \mid 20$ concepts (submitted respectively to the M3 and M4 ESA Calls) also rely on a TMA derived telescope, enforcing the concepts of differential measurement of fields close to the Sun, affected by fairly large light deflection, with a dedicated design deployed around a coronagraphic subsystem. 
Table 1. Main design parameters

$\begin{array}{ll}\text { Telescope diameter } & 1 \mathrm{~m} \\ \text { Focal length } & E F L=15 \mathrm{~m} \\ \text { Spectral range } & \text { Visible, } \lambda=0.55 \mu \mathrm{m} \\ \text { Spectral band } & \Delta \lambda=200 \mathrm{~nm} \\ \text { Field of view } & \text { Annular } \\ \text { FOV radius } & \theta \simeq 1^{\circ} \\ \text { Detector } & \mathrm{sCMOS} \text { or CCD } \\ \text { Pixel size } & 4 \mu \mathrm{m}\end{array}$

Table 2. Optical design parameters

\begin{tabular}{l|r|r|r} 
Mirror & $\begin{array}{r}\text { Curvature } \\
\text { radius [mm] }\end{array}$ & $\begin{array}{r}\text { Distance } \\
{[\mathrm{mm}]}\end{array}$ & $\begin{array}{r}\text { Conic } \\
\text { constant }\end{array}$ \\
\hline M1 & $3,500.00$ & $1,400.00$ & -0.98 \\
M2 & 859.45 & $2,800.00$ & -1.93 \\
M3 & $1,121.55$ & $1,452.65$ & -0.58
\end{tabular}

TMAs may achieve extremely good performance, but they also feature comparably high sensitivity to misalignment,21 as understandable from their large étendue (product of aperture area and field angular coverage). The annular field telescope proposed in this paper actually achieves an intermediate étendue, since the field of view range is large, but not filled, i.e. only a fraction of the subtended phase space is actually used.

A typical TMA feature is a significant vignetting of the central region, so that they are often used in an off-axis configuration; e.g., JWST is on-axis in aperture and off-axis in field. Our proposed annular field can be considered as an off-axis case, replicated by symmetry around the optical axis (resulting in an on-axis telescope that uses only a corona region of focal plane); ring field telescopes have been proposed e.g. for Earth observation, $\stackrel{22]}{\text { with a }}$ thorough discussion of the design approach and performance. Actually, an astronomical telescope with annular field was proposed for the DUNE concept, 23 but this design feature aspect was dropped when it was merged with the SPACE proposal in the Euclid space mission.24

\subsection{Science specifications}

The main performance driver of our study is astrometric accuracy in the determination of angular distance between bright, unresolved sky objects. We set our reference magnitude in the range $8-10 \mathrm{mag}$ at visible wavelengths, for near-solar type stars. The observing wavelength is set in the visible: $\lambda \simeq 0.55 \mu m$, with a fairly large spectral bandwidth $\Delta \lambda \simeq 200 \mathrm{~nm}$. The number of photons per hour received from a $D=1 \mathrm{~m}$ diameter telescope, with a $0.6 \mathrm{~m}$ central obstruction (case explored in our study, see below), ranges between $\sim 3.6 e 9$ $(10 \mathrm{mag})$ to $\sim 2.3 \mathrm{e} 10(8 \mathrm{mag})$.

This is expected to be compatible with the precision goal, assuming repeated visits to each target throughout a mission lifetime of $\sim 5$ years, and a $1 \mathrm{~m}$ class telescope operating close to the diffraction limit. The angular resolution, corresponding to the characteristic image size, is $\sim \lambda / D \simeq 110$ mas.

The photon limited precision on location in 1 hour of observation, in each coordinate, is $\sim 2 \mu \mathrm{as}$ for a $10 \mathrm{mag}$ unresolved source. In any case, the observation is assumed to be split in a sequence of shorter exposures, limited by detector saturation to a few or several $10^{4}$ photo-electrons. This corresponds to an elementary exposure time of order of a few $10 \mathrm{~ms}$, feasible by acquisition of selected regions of interest, if not by full frame readout.

Astrometric uncertainty scales with photons to $0.7 \mu a s$ ( $8 \mathrm{mag})$; moreover, the nominal location dispersion (and similarly the precision on related parameters, e.g. parallax or orbit semi-axis) scales with the square root of exposure time (e.g. by a factor 4.9 in 24 hours) and of the number of visits $N_{V}$, so that sub- $\mu a s$ performance is achievable, in an ideal world. In practice, it is usually considered unavoidable to hit the "floor" imposed by instrument perturbations and, ultimately, calibration uncertainties.

One of the main drivers of our study is the definition of a measurement system featuring not only high astrometric precision, but above all suited to good calibration, both from sky measurement and metrology.

The most relevant specifications for the instrument outlined in this study are summarized in Tab. 1, and further expanded in the following sections. 


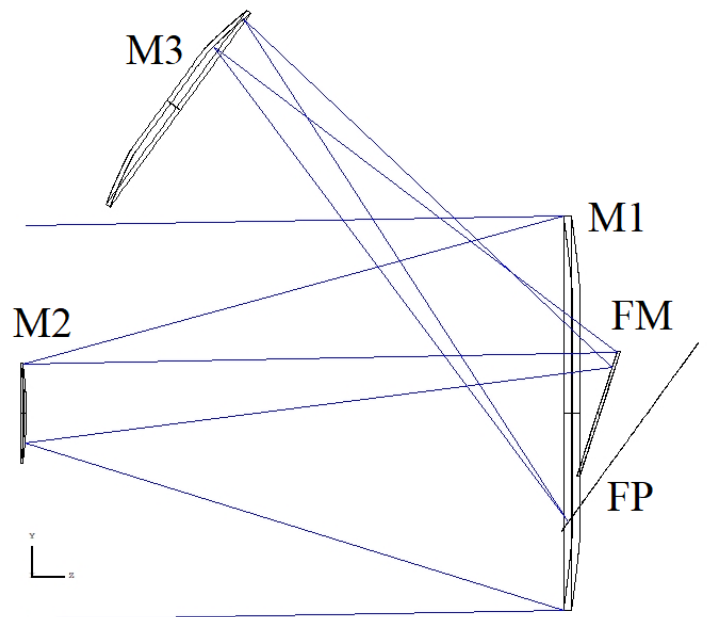

Figure 2. Telescope layout 2D, bent configuration.

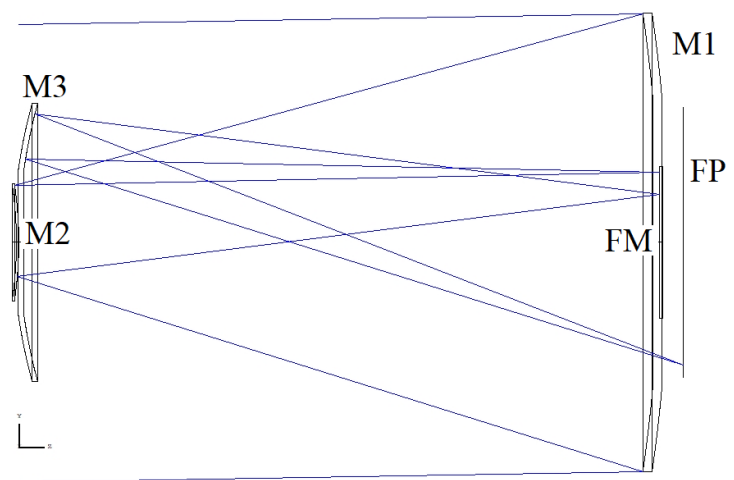

Figure 3. Telescope layout 2D, on-axis, centered configuration.

\subsection{Optical Design Deployment}

We aim at a $1 \mathrm{~m}$ class telescope with intrinsically good astrometric response on an annular field of view with $1^{\circ}$ radius. An additional asset for a space instrument is of course a compact envelope, which is expected to be conducive to a better trade-off between mass budget and mechanical stability.

For convenience, we adopt an example configuration from the literature $\stackrel{25}{, 25}$ and our optical design specifications are implemented by adaptation of Solution IV in that paper. The improvements consist in increasing the aperture and reducing the EFL to the desired values, and above all modifying the layout with the goal of a very compact configuration, as will be shown in Fig. 33. The optical design modelling, ray tracing and optimization is performed with Zemax. The main optical parameters are summarized in Tab. 2.

A TMA often folds the configuration over two axes by introducing a flat mirror between secondary and tertiary (curved) mirrors, in order to deploy the optical components in separated, easily accessible regions, usually "behind" the primary mirror. Our proposed design folds the optical axis back onto itself, in order to minimize the overall instrument volume; as this only requires to turn a (flat) folding mirror, it is trivial from the standpoint of optical design. However, this allows trade-offs among different geometry constraints (related to vignetting and mutual obscuration), which are nonetheless compatible with our goal of an annular field of view.

In Figs.22 and 3, two layout options of the same underlying optical design are proposed; they are equivalent, since curved mirrors have the same shape and separation, at first order, but they are placed in different positions. On the left panel, the tertiary mirror is placed on the side of the input beam, whereas on the right panel it is located on-axis, close to the secondary mirror. In the former case the optical path is easier to follow, whereas the latter is more compact, and it is our preferred option. Their relative merits will be discussed in Sec.3.

In either figure, the converging beam from the primary mirror (M1, right end side) reaches the secondary mirror (M2, left end side), then proceeds to the flat folding mirror (FM) located close to the central area of M1. The beam is then reflected back to the tertiary mirror (M3), located in proximity of M2, which focuses it onto the ring of detectors of the focal plane (FP), again placed approximately in the intermediate region between FM and M1.

A three-dimensional representation of the instrument is shown in Fig.4. Due to the annular field, with the selected parameters, no mutual vignetting exists among M1, M2, FM and M3.

\subsection{Optical performance}

The optical performance results in diffraction limited imaging over the whole annular region between radial values $0.9^{\circ}$ and $1.1^{\circ}$, so that the corrected field exceeds $\sim 2 \pi \times 0.2=1.256$ square degrees. 


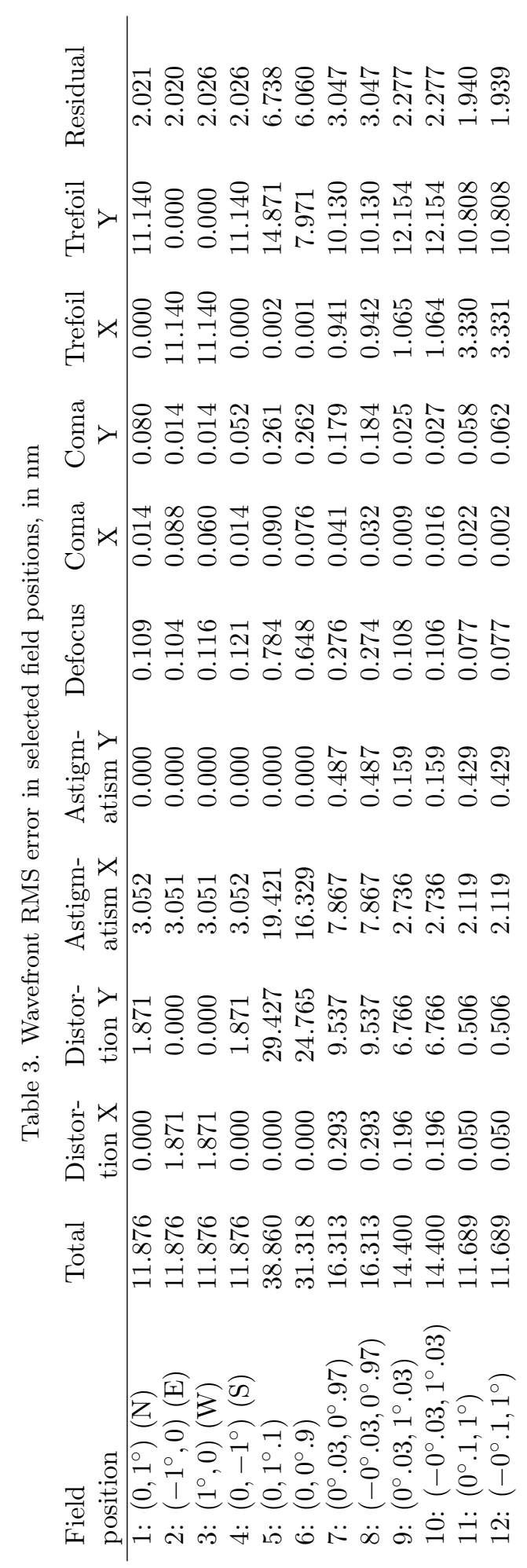




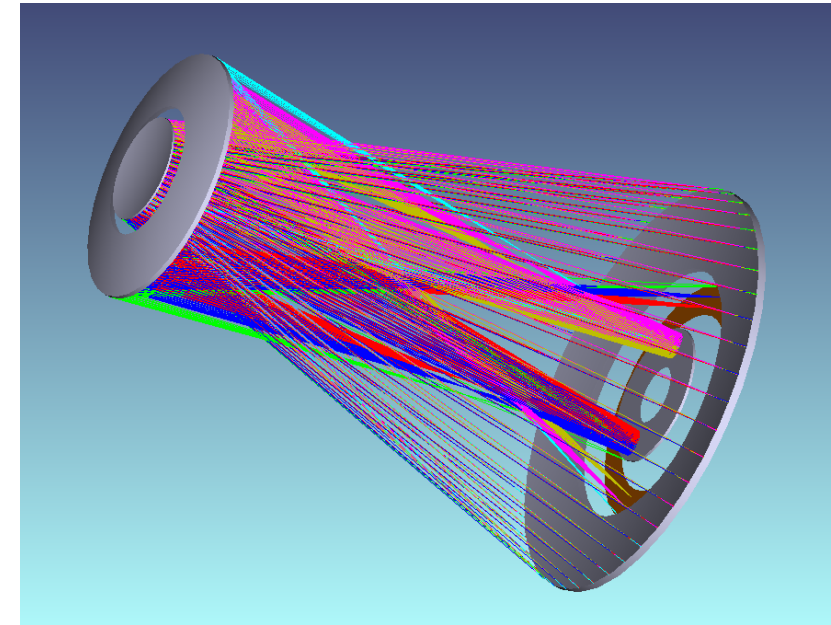

Figure 4. Telescope 3D view, layout A: on-axis configuration

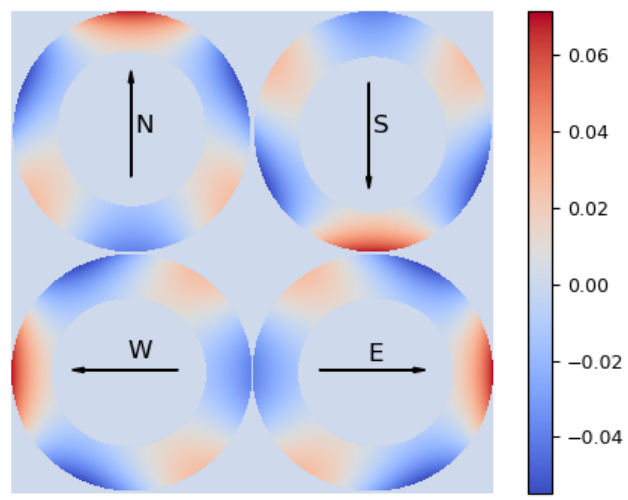

Figure 6. Wavefront error maps, in waves at $550 \mathrm{~nm}$ at $1^{\circ}$, at four equally spaced positions (fields 1, 2, 3 and 4 in Tab. 3 along the ring at $1^{\circ}$ from the optical axis.

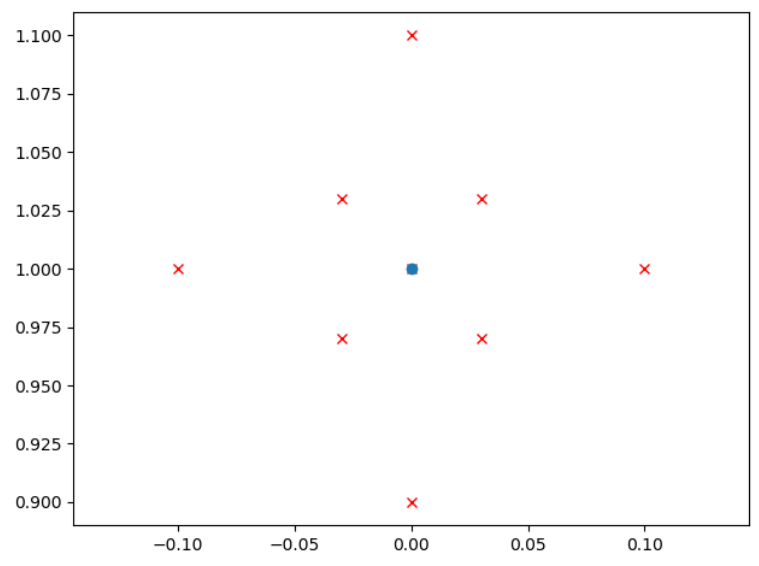

Figure 5. Field positions selected for analysis around the reference position $\left(0,1^{\circ}\right)$.

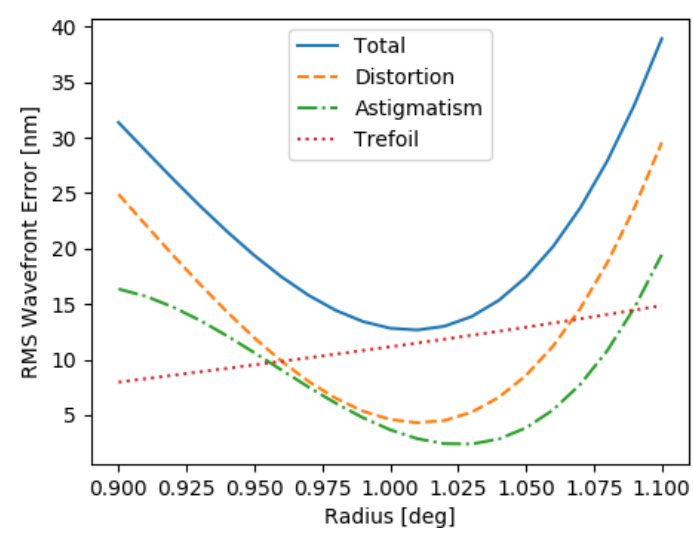

Figure 7. RMS wavefront error and its main components vs. radial coordinate.

The maps have a clear structure with three periods around the pupil, corresponding to trefoil in terms of classical aberrations (Zernike polynomials $n=3, m= \pm 3$ ). This is actually the dominant term along the $1^{\circ}$ ring, accounting for most of of RMS WFE $11.14 \mathrm{~nm}$. Small amounts of distortion $(1.87 \mathrm{~nm})$ and astigmatism $(3 \mathrm{~nm})$ are also present, with a residual of $2 \mathrm{~nm}$. It may be noted that trefoil, given its symmetry, is expected to induce negligible systematic astrometric errors in the azimuthal direction.26 The dominant trefoil results in the trilobated structure of the PSF (Fig.8), particularly evident in the first diffraction ring.

In Tab. 3, $\mathrm{X}$ and $\mathrm{Y}$ are angular coordinates on the tangent plane, corresponding respectively to radial and azimuthal directions for field 1 . The radial coordinate is reversed in field 4 , and aligned with $\pm Y$ axis in fields 3 and 2, respectively. The circular symmetry is evidenced e.g. by the exchange of $\mathrm{X}$ and $\mathrm{Y}$ components of distortion and trefoil. The WFE analysis yields the same results for all fields over the $1^{\circ}$ radius circle, in the local frame of radial and azimuthal coordinates.

Field positions 5 to 12, describing a small area around the reference field, and shown in Fig. 5 , correspond to the size of one detector ( 7 to 10 ), and to the radial size of the corrected field.

The annular region $\left(0.9^{\circ}<\right.$ radius $\left.<1.1^{\circ}\right)$ is limited by increasing values of mainly astigmatism and distortion; actually, the latter does not degrade imaging quality, but only affects astrometric error, exclusively in the radial direction. Not including distortion, the RMS WFE at the ring borders is still $\sim 25 \mathrm{~nm}$, i.e. better than $1 / 20$ wavelengths at $550 \mathrm{~nm}$. 

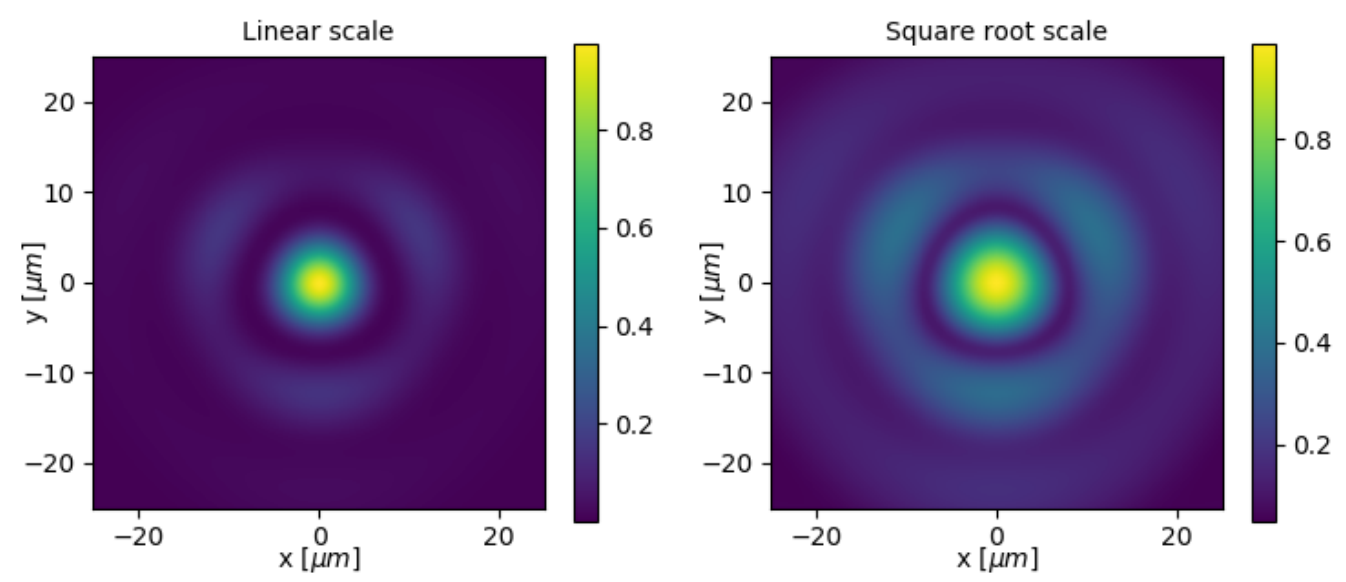

Figure 8. Polychromatic PSF in field $1,1^{\circ} \mathrm{N}$, in linear (left) and square root (right) scale.

The RMS WFE and its main components (distortion, astigmatism and trefoil) are shown in Fig. 7 as a function of the radial distance from the optical axis. The minimum value is close to $1^{\circ}$, but the aberration balancing is not exactly coincident for all components. The PSF at $1^{\circ}$, produced by a near-Solar unresolved source, using a 20\% spectral bandwidth (approximating a full band, filter-less instrument), is shown in Fig. 8 on a linear (left) and square root (right) scale. The first diffraction ring around the central lobe is remarkably brighter than that of a conventional filled circular aperture, due to the large central obstruction.

The PSF is symmetric (zero skewness) with respect to the radial axis, due to the underlying circular symmetry of the system; aberrations introduce small asymmetric contributions only along the radial direction (skewness: 0.2 ), i.e. with respect to the azimuthal axis. The resulting correlation between components of the photocentre estimate, according to the mathematical framework detailed in the Appendix, is therefore expected to be negligible. Numerical tests, performed in high SNR conditions, actually provide results for the Pearson correlation coefficient in the range $10^{-15}$ to $10^{-18}$.

\section{DISCUSSION}

Moreover, modern optical design tools are able to achieve optimal ray tracing solutions using quite complex userdefined strategies. It is thus possible to require good performance over the selected ring of the focal plane, even at the expense of penalties on image quality at the centre of the field. This contrasts with "old fashioned" analytical approaches pre-dating recent developments in computing power, in the epoch (very few decades ago!) mainly based on perturbation theory. In such context, on-axis performance was typically the main criterion for the initial design step, then third order aberrations were considered, and successively higher terms were introduced (fifth order), in an attempt to achieve satisfactory optical performance by aberration balancing. The technique is not superseded by modern ray tracing, which relies heavily on it, and just takes advantage of numerical "brute force" to explore a much larger parameter space than suited to direct human investigation.

Conventional optical designs based on the requirement of a large field of view usually allocate it to a large contiguous region (square, circular, hexagonal or other) around the optical axis. The ray tracing optimization process achieves sufficient image quality over the overall field, usually with degradation increasing with the distance from the axis itself, where usually the response is (nearly) diffraction limited. Spectroscopic instruments may not have actual image quality requirements, but their performance is nonetheless based on limited wavefront error (WFE) over the field, e.g. to ensure adequate matching of point-like sources to the slit or fiber feeding the dispersing system.

The FOV requirement is sometimes actually related to a contiguous area, e.g. for imaging large sky regions, e.g. Solar System planets, the Moon, globular clusters and so on, but it may be relaxed if the science needs can 
Table 4. Astrometric error vs. azimuthal field position, using Maximum Likelihood estimator and the central PSF as reference, in the radial and azimuthal direction, either ignoring or taking into account the local field rotation.

\begin{tabular}{lccc}
$\begin{array}{l}\text { Field } \\
\text { position }\end{array}$ & $\begin{array}{c}\text { Radial } \\
{[\mathrm{mas}]}\end{array}$ & $\begin{array}{c}\text { Azimuthal } \\
\text { Non Rotated } \\
{[\mu a s]}\end{array}$ & $\begin{array}{c}\text { Azimuthal } \\
\text { Rotated } \\
{[\mu a s]}\end{array}$ \\
\hline 1: $\left(0,1^{\circ}\right)(\mathrm{N})$ & 0.0000 & 0.0000 & 0.0000 \\
2: $\left(-1^{\circ}, 0\right)(\mathrm{E})$ & 0.0004992 & $3.592 \mathrm{e}-12$ & 0.0000 \\
3: $\left(1^{\circ}, 0\right)(\mathrm{W})$ & -0.01504 & $4.131 \mathrm{e}-12$ & 0.0000 \\
4: $\left(0,-1^{\circ}\right)(\mathrm{S})$ & -0.01554 & $-3.709 \mathrm{e}-12$ & 0.0000 \\
5: $\left(0,1^{\circ} .1\right)$ & -0.8184 & $-5.325 \mathrm{e}-12$ & $-5.325 \mathrm{e}-12$ \\
6: $\left(0,0^{\circ} .9\right)$ & 0.7836 & $-3.853 \mathrm{e}-12$ & $-3.853 \mathrm{e}-12$ \\
7: $\left(0^{\circ} .03,0^{\circ} .97\right)$ & 0.2198 & -39.72 & -0.0028 \\
8: $\left(-0^{\circ} .03,0^{\circ} .97\right)$ & 0.2198 & 39.72 & 0.0003 \\
9: $\left(0^{\circ} .03,1^{\circ} .03\right)$ & -0.2279 & -16.3 & 0.0015 \\
10: $\left(-0^{\circ} .03,1^{\circ} .03\right)$ & -0.2279 & 16.3 & 0.0001 \\
$11:\left(0^{\circ} .1,1^{\circ}\right)$ & -0.0125 & -82.85 & 0.0010 \\
$12:\left(-0^{\circ} .1,1^{\circ}\right)$ & -0.0125 & 82.85 & -0.0024
\end{tabular}

be expressed just in terms of achieving simultaneous measurement of a sufficiently large area to ensure adequate source statistics, e.g. availability of many stars above a given magnitude threshold. In particular, exo-planet astrometry requires determination of the distance between a bright target with respect to a reference source of comparable magnitude, or several sources not much fainter. Since the number of bright stars is limited, a fairly large accessible FOV is considered as a crucial aspect.

\subsection{Exploitation of image symmetry}

The concept of symmetry is intrinsically appealing, but it is necessary to identify practical tools for assessment of the degree of success toward our goals. Every detector of the focal plane covers the same small range of angular positions with respect to the optical axis, since they are all interchangeable by a simple rotation of the overall system. This is a crucial element of the planned calibration principle. Also, in each device, the main directions of the pixel array (rows and columns) can in principle be aligned with local radial and azimuthal axes.

In the nominal system, residual aberrations can be expected to be characterized by well defined symmetry; most of them, e.g. spherical, astigmatism, trefoil, distortion and coma, are symmetric in the azimuthal direction, although some are not along the radial direction. Therefore, the PSF is symmetric in the azimuthal direction, and at most it can have asymmetric terms in the radial direction. As derived in the appendix, this means that the photo-centre estimates in the two directions is not correlated, and this is a crucial property with respect to control of systematic astrometric errors.

It can be expected that, within the limit of small perturbations, the image symmetry properties will be retained, provided the real systems are not exceedingly removed from the nominal symmetry conditions. The issue can be addressed with standard tools of advanced optical design, in particular tolerancing analysis.

For the set of field positions considered in Tab. 3, the reconstructed PSF photo-centre is computed, using a Maximum Likelihood estimator ${ }^{927}$ and the central position PSF (coordinates $\left(0,1^{\circ}\right)$ ) as reference. The systematic error, i.e. the PSF displacement with respect to the template, is listed in Tab. 4, and (for field positions 5 to 12) shown in Fig.9. We remark that values are reported in mas for the radial coordinate, and in uas for the azimuthal coordinate.

Field positions 2, 3 and 4 provide displacement close to zero with respect to the template, apart the natural rotation of the local reference system, due to symmetry reasons. The discrepancy with respect to mathematical zero is considered to be originated by sampling and numerical approximations; it is larger (but still a small fraction of 1 mas!) in the radial direction, which does not benefit from symmetry, and much smaller in the azimuthal direction.

In a local region around the reference $\mathrm{N}$ position (fields 5 to 12), the location estimate provides radial errors below 1 mas, and azimuthal errors within a few $10 \mu a s$, if the local rotation of the PSF is not taken into account; 


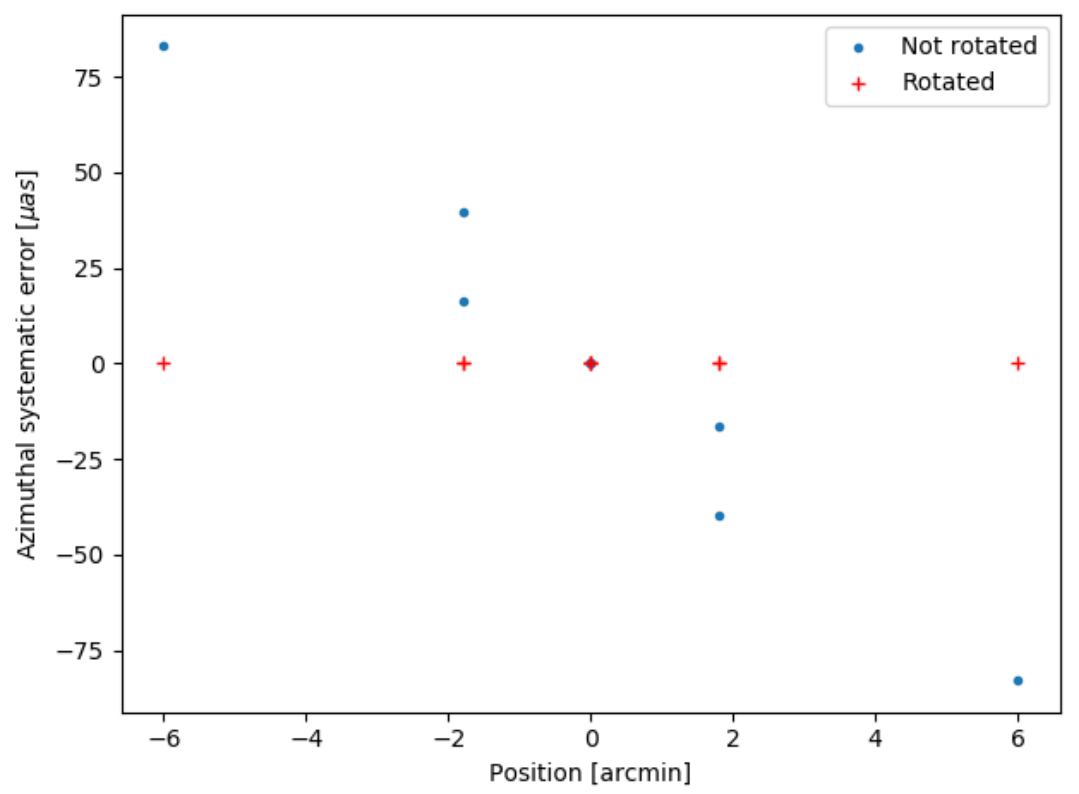

Figure 9. Astrometric error vs. azimuthal field position, using Maximum Likelihood estimator and the central PSF as reference, in the radial and azimuthal direction; dots: field rotation not considered; crosses: field rotation included.

when properly including such geometric factor, the azimuthal error drops to the few nano-arcsec range (last column of Tab. 4, which appears consistent with the model limitations.

Therefore, the nominal RAFTER configuration appears particularly well behaved with respect to robust astrometric measurement, above all along the azimuthal direction, over the whole 1.256 square degrees corrected field.

\subsection{Engineering aspects}

The telescope layout depicted in Figs. 2 and 3 is proposed in two options. In the former, the optical path is bent to get out M3 of the input beam. In the latter, the folding mirror reflects the optical axis back onto itself, placing the tertiary mirror M3 close to the secondary mirror M2. Although in the former (labelled "B" for "Bent") the beam path is easier to follow, we reckon that the latter (labelled "A" for "on-Axis") is worth taking into account because of its compactness. The drawback of layout A consists of course in the large central obscuration of the beam, traded off in exchange for the compact layout. The rationale is not only associated to efficient usage of the precious volume of a space mission payload, but rather in the expected advantages in terms of higher stiffness and/or lower mass budget of the system.

From the strictly optical standpoint, layout A suffers from lower throughput than B, because the central obscuration increases from $\sim 30 \%$ to $\sim 60 \%$ in linear terms, resulting in a collecting area penalty of $\sim 9 \%$ and $\sim 36 \%$ respectively. This holds under the assumption that the primary mirror diameter is retained to the same value ( $1 \mathrm{~m}$ in our case). However, this is not necessarily the only way to compare the two options. If we set constraints on the overall volume or mass budget, rather than on M1 diameter, the balance shifts, and it is less obviously in favour to option B.

In particular, with fixed total mass, layout A is compatible with a larger M1 diameter (M1 is intrinsically lightweighted by the larger central obstruction), thus recovering at least part of the collecting area, and improving on the imaging resolution. If we set equal M1 area, i.e. matching photon budget, layout A requires a primary diameter $D_{1}=1.2 \mathrm{~m}$, and provides $20 \%$ better resolution, with marginal changes in the remaining parts of the optical system. An equal astrometric performance criterion would result in a M1 diameter $D_{1} \simeq 1.1 \mathrm{~m}$.

The on-axis layout A benefits from a further asset, due to the naturally grouping of optical components in two subsets, including respectively M2 and M3 (left side in figure), and M1, FM and FP (right side), with 
Table 5. Detailed report of the Zemax sensitivity analysis focused on the most critical parameters ("offenders"). Analysis conducted for a representative wavelength of $632.8 \mathrm{~nm}$. Optical elements labels are reported in Figure 3 Z axis is the optical axis. All parameters not listed give negligible contribution.

\begin{tabular}{cccc} 
Element & Parameter & Variation & Change \\
\hline M1 & Tilt X and Y & $+/-3 '$ & 21.44 \\
M2 & Tilt X and Y & $+/-3 '$ & 5.48 \\
M1 & Movement X and Y & $+/-0.2 \mu m$ & 2.27 \\
M2 & Movement X and Y & $+/-0.2 \mu m$ & 2.24 \\
FM & Tilt X and Y & $+/-3 '$ & 1.97 \\
M1 - M2 & Distance & $+/-0.2 \mu m$ & 1.78 \\
M3 & Tilt X and Y & $+/-3 '$ & 0.47 \\
M1 & Surface irregularity & +0.2 Fringes & 0.46 \\
M2 & Surface irregularity & -0.2 Fringes & 0.22 \\
M1 & Radius & -1 Fringe & 0.13 \\
M2 & Radius & +1 Fringe & 0.09 \\
M2 - FM & Distance & $+0.2 \mu m$ & 0.04 \\
M3 & Movement X and Y & $+/-0.2 \mu m$ & 0.02
\end{tabular}

a significant separation between them. The two groups of component are faced to each other, and might in principle be connected by a stable structure (e.g. a cylindrical Serrurier-like truss).

It is possible to envisage the manufacturing of M2 and M3 from a single blank, e.g. by diamond turning, or their mounting on a common bench, e.g. by optical contact bonding. This would ensure a very stable geometry of the subsystem, established at manufacturing stage; the actual optical alignment between M2 and M3 then depends exclusively on FM, as its tilt may perturb, or correct, the coincidence between individual optical axes. The M1, FM and FP subset can also be mounted on an optical bench (e.g. a Gaia-like torus, but much smaller), to achieve good mutual stability. Thus, the optical configuration would be preserved by mutual alignment of the two sub-systems, faced to each other. This does not necessarily alleviates all mechanical stability requirements, but at least it provides a simple geometric framework.

In layout B (Fig.2, lower right), some of the sensors are placed closer to M1 and FM than others, and could be suppressed if necessary, whereas layout A benefits of a more uniform geometry, which is expected to be beneficial to the overall system symmetry and minimization of systematic errors.

The configuration optimization is anyway left to future, more detailed development phases. In particular, aberration balancing (Fig.7) may be improved, especially on distortion. However, the corrected field already exceeds $\sim 2 \pi \times 0.2=1.256$ square degrees. Since a much smaller area $(0.258$ square degs $)$ in the midst of the ring field is actually populated with detectors, on account of cost and complexity, there are significant margins against perturbations degrading the optical quality, e.g. misalignment, since external areas are expected to be more sensitive to them.

\subsection{Sensitivity to misalignment}

Conventional sensitivity analysis is usually aimed at finding the linear and angular ranges beyond which the telescope imaging quality (or other suitable descriptors) suffers from excessive degradation. It can be shown that the proposed configuration is, in this respect, quite comparable to other TMAs with similar design parameters (aperture diameter, effective focal length, etc.), while providing, as discussed above, potential benefits toward ease of implementation of a compact, stable mechanical assembly.

Tab. 5 reports the results of the preliminary sensitivity analysis conducted with the traditional techniques. We analyzed the position of the elements and their tilting, as well as the surface irregularities. The system has an axial symmetry that results in an equivalence of the tilts and displacements with respect to the $\mathrm{X}$ and $\mathrm{Y}$ axis. The M1 and M2 tilts are the most offending parameters, while all other variations, included the separations and the surface irregularities are well within an acceptable level. 


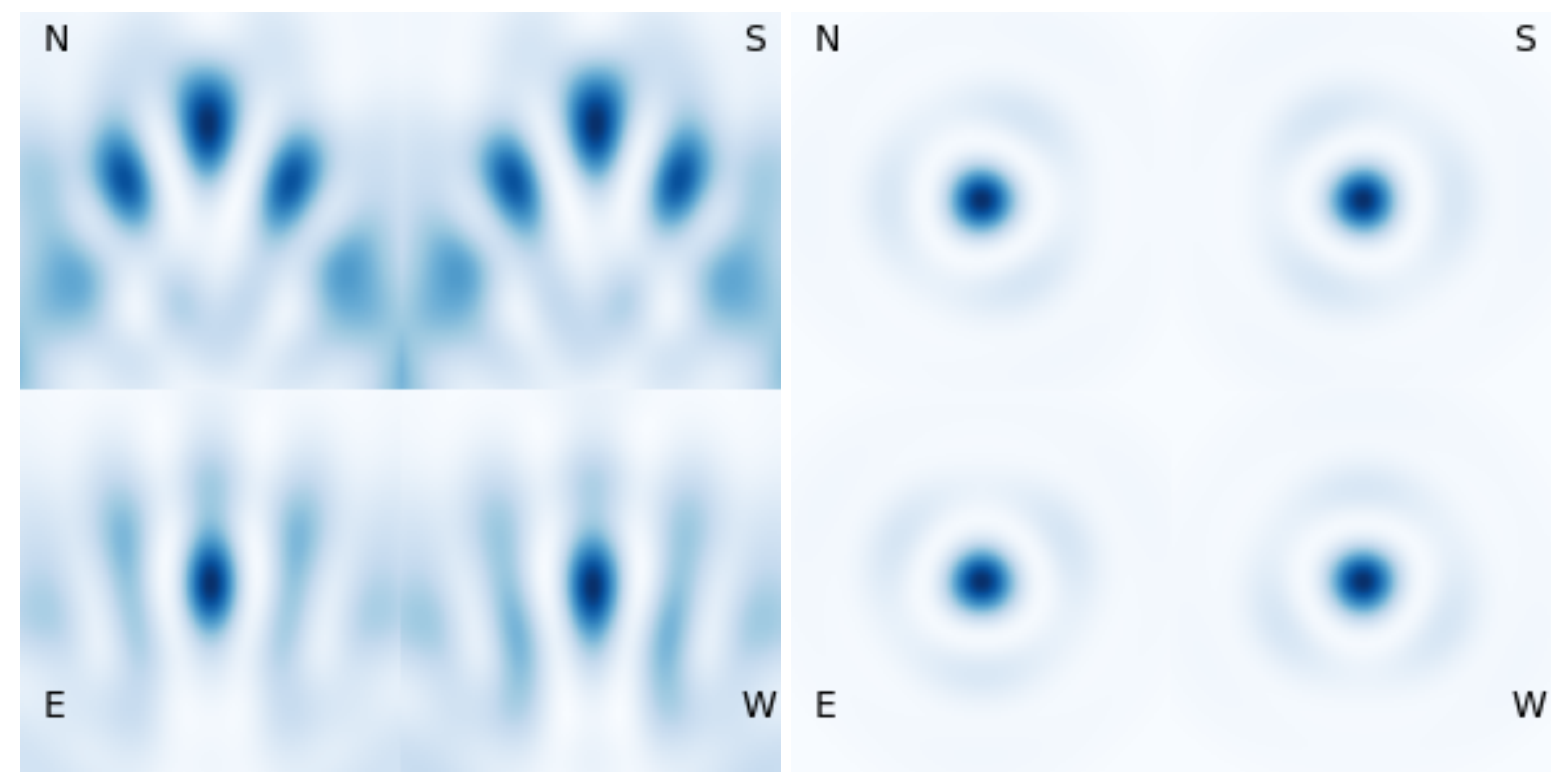

Figure 10. PSF degradation for large (36 arcsec, left) and medium (1 arcsec, right) tilt of M1.
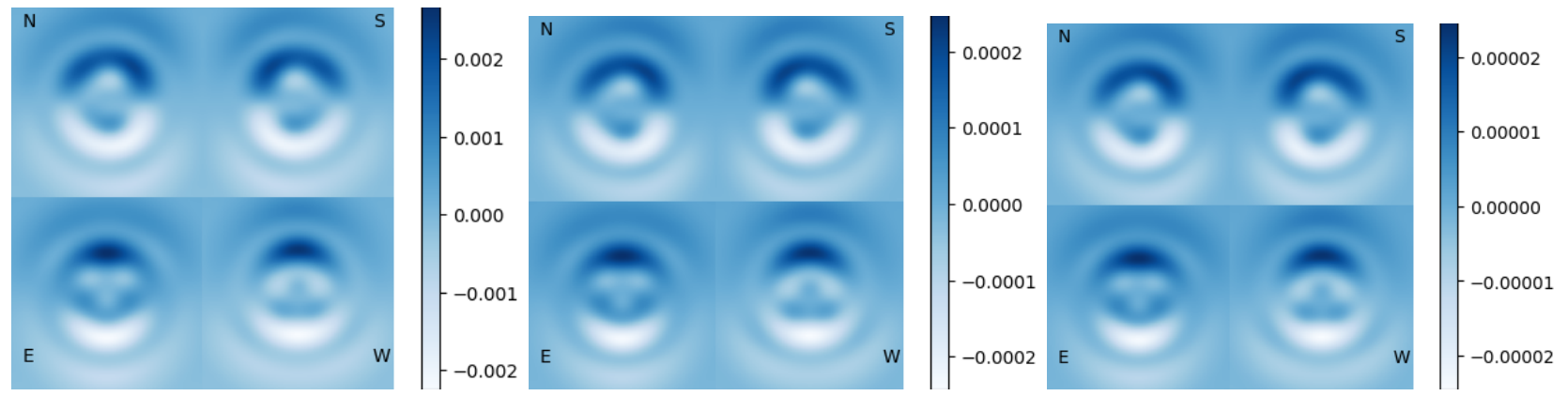

Figure 11. PSF variation for medium (1 arcsec, left), small (100 mas, center) and tiny (10 mas, right) tilt of M1.

Limiting values for M1 tilt are in the few ten arcsec range, depending on the actual threshold on the figure of merit, while all other components are retained in the nominal position; an example of PSF generated in field positions 1 to 4 (labelled respectively N, E, W and S) of Tab. 3 is shown in Fig.10 (left), evidencing a strong degradation of the diffraction limited image, for a 36 arcsec tilt of M1. Smaller perturbations generate images very similar to the nominal case, as shown in Fig.10 (right) for a 1 arcsec tilt of M1. The M1 tilt direction is N-S. vertical in figure.

Actually, the effect is more clearly evidenced by the image difference with respect to the nominal PSF in the same field position, as shown in Fig. 11 for a tilt of M1 by 1 arcsec (left), 100 mas (centre) and 10 mas (right), respectively. As it might be expected, the graphical aspect of the PSF shape change is similar, and corresponding to what it could be expected from a tilt, i.e. image displacement, but the amplitude is clearly different, scaling approximately as the applied tilt. The PSF variation is quite similar in the four field positions, carrying the hallmark of the applied tilt, and therefore it introduces perturbations to the rotational PSF symmetry over the field with a clear azimuthal dependence. Since the PSF is computed around a nominal field position corresponding to the gnomonic projection of the angular position in the object space, the net effect appears to be an "excess tilt".

The annular field of RAFTER provides therefore a convenient leverage toward identification of aberrations, thanks to their field dependence, contrarily to cases discussed in the literature according to which TMAs are difficult to align, because the situation taken into account was based on a single sensor (e.g. a WFS) in a fixed 
Table 6. Astrometric error, in mas, originated by a M1 tilt in field positions 1 to 4, labelled N, S, E and W.

$\begin{array}{lcccc}\text { M1 tilt [deg] } & 3 e-6 & 3 e-5 & 3 e-4 & 1 e .3 \\ \text { N, radial } & -0.001289 & -0.01288 & -0.1283 & 37.4 \\ \text { N, azimuthal } & 8.223 \mathrm{e}-15 & -2.366 \mathrm{e}-16 & 5.236 \mathrm{e}-16 & -5.345 \mathrm{e}-13 \\ \text { S, radial } & -0.001302 & -0.01303 & -0.1311 & 36.67 \\ \text { S, azimuthal } & -7.162 \mathrm{e}-16 & 2.035 \mathrm{e}-15 & 2.262 \mathrm{e}-16 & -3.973 \mathrm{e}-13 \\ \text { E, radial } & -7.825 \mathrm{e}-08 & -7.643 \mathrm{e}-06 & -0.0007641 & -2.543 \\ \text { E, azimuthal } & -0.001097 & -0.01097 & -0.1098 & 34.99 \\ \text { W, radial } & 7.824 \mathrm{e}-08 & 7.641 \mathrm{e}-06 & 0.000764 & 2.544 \\ \text { W, azimuthal } & -0.001097 & -0.01097 & -0.1098 & 34.99\end{array}$
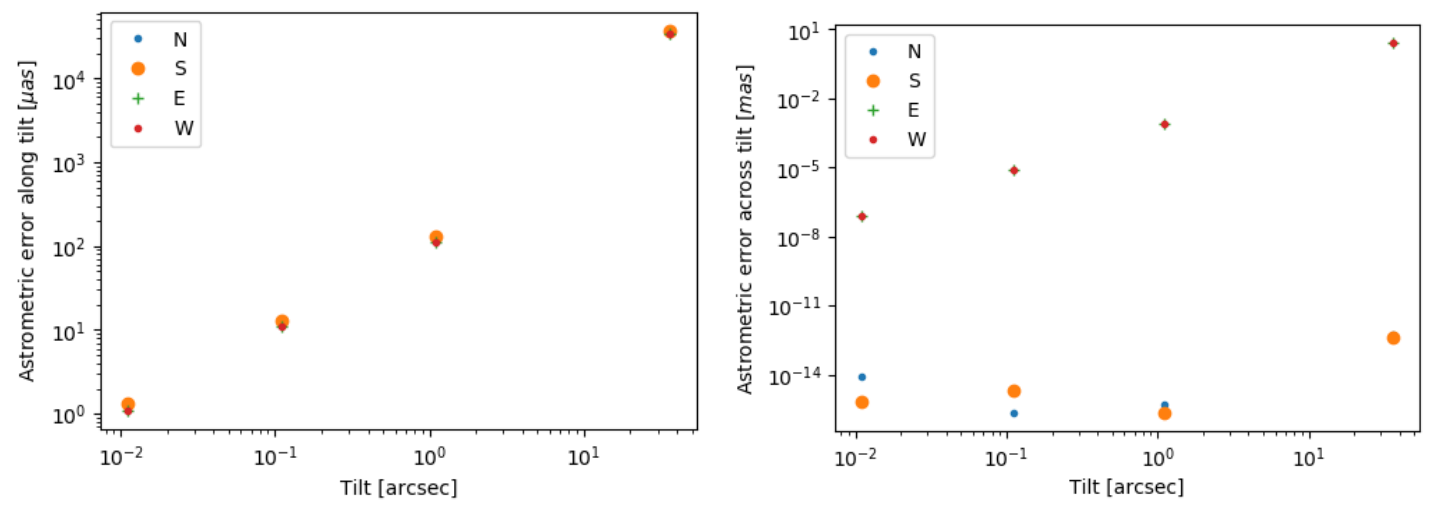

Figure 12. Astrometric error originated by a M1 tilt in field positions 1 to 4, respectively along (left) and across (right) the tilt direction.

field position.

By application of the Maximum Likelihood estimator, and the local nominal PSF as reference, we compute the photo-center displacement induced by the M1 tilt. The results are listed in Tab. 6, and shown in Fig.12, respectively for the direction aligned with the applied tilt (left panel), and orthogonally to it (right panel). Notably, the tilt direction is radial for fields $\mathrm{N}$ and $\mathrm{S}$, and azimuthal for fields $\mathrm{E}$ and $\mathrm{W}$.

Small perturbations, about one order of magnitude below critical values evidenced by the optical sensitivity analysis (Tab. 5), are associated to a linear range of astrometric disturbances, in this case mostly aligned with the tilt direction. The astrometric sensitivity to M1 tilt is $\sim 0.1 \mathrm{mas} / \mathrm{arcsec}$, i.e. the effect is reduced by approximately four orders of magnitude. Across-tilt effects are much smaller. Moreover, the astrometric error associated to M1 tilt is common mode to the whole annular focal plane, so that it is compensated to a high degree in differential measurements between sources along the ring: the focal plane separation is mostly insensitive to the instrument perturbation.

\section{CONCLUSIONS}

We propose an innovative solution to the challenging problem of achieving a highly uniform optical response over a large field of view. The design driver is the achievement of an annular field of view around the optical axis of the telescope, with symmetry preserved to a large extent. The proposed design is a very compact, $1 \mathrm{~m}$ class telescope compatible with modern CCD and CMOS detectors, with effective focal length EFL $=15 \mathrm{~m}$, based on the established TMA telescope concept. We describe the concept implementation guidelines and the optical performance of the current configuration. The layout is extremely compact with respect to comparable TMA telescopes, since it is mostly deployed along the main optical axis. Circular symmetry is preserved for each optical element and for the overall system. The proposed design is considered as representative of a family of telescopes with the same symmetry. The diffraction limited FOV exceeds 1.25 square degrees, and in the proposed version 
the detector takes the best 0.25 square deg, using 66 devices. The detector is positioned close to the primary mirror for compactness, and easily interfaced to a radiator on the external envelope of the payload.

The design is easily scalable and suited to be tailored to the needs of a range of space missions based on medium size telescopes and aiming at photon limited astrometric precision in the few $\mu a s$ range. An example, with two configurations featuring respectively $1 \mathrm{~m}$ and $0.7 \mathrm{~m}$ primary diameter, is provided in another contribution to these Proceedings for the AGP mission concept.

The good astrometric performance of RAFTER fulfills several of the goals settled in the ASTRA study, also presented in these Proceedings. Future developments may be devoted to further improvement of the optical performance uniformity over larger fields, optimization of an embedded metrology system, and inclusion of a multiple LOS beam combiner for large spherical angle astrometry.

\section{ACKNOWLEDGMENTS}

The INAF activity has been partially funded by a grant from the Italian Ministry of Foreign Affairs and International Cooperation, and by the Italian Space Agency (ASI) under contracts 2014-025-R.1.2015 and 2018-24-HH.0.

\section{REFERENCES}

1. M. Shao, S. G. Turyshev, C. Zhai, et al., "Finding Exo-Earths with Precision Space Astrometry," Bull. American Astron. Soc. 51, p. 74, May 2019.

2. F. Malbet, A. Léger, M. Shao, R. Goullioud, et al., "High precision astrometry mission for the detection and characterization of nearby habitable planetary systems with the Nearby Earth Astrometric Telescope (NEAT)," Experimental Astronomy 34, pp. 385-413, Oct. 2012.

3. F. Malbet, A. Léger, G. Anglada Escudé, A. Sozzetti, et al., "Microarcsecond astrometric observatory Theia: from dark matter to compact objects and nearby earths," in Proc. SPIE, Society of Photo-Optical Instrumentation Engineers (SPIE) Conference Series 9904, p. 99042F, July 2016.

4. E. Bendek, P. Tuthill, O. Guyon, et al., "Precision astrometry mission for exoplanet detection around binary stars," in Proc. SPIE, Society of Photo-Optical Instrumentation Engineers (SPIE) Conference Series 10698, p. 106980G, Aug. 2018.

5. I. Hahn, M. Shao, and S. G. Turyshev, "Microarcsecond Astrometry Telescope on the DSG," in Deep Space Gateway Concept Science Workshop, 2063, p. 3015, Feb. 2018.

6. M. Gai, Z. Qi, M. G. Lattanzi, B. Bucciarelli, D. Busonero, M. Crosta, F. Landini, H. Luo, S. Liao, G. Mana, R. A. Méndez, M. Pisani, A. Riva, C. I. J. San Martin Luque, C. P. Sasso, Z. Tang, A. Vecchiato, and Y. Yu, "The ASTRA project: a doorway to future astrometry," in This Conference, SPIE Conference Series, 2020.

7. L. Lindegren, "Photoelectric astrometry - A comparison of methods for precise image location," in $I A U$ Colloq. 48: Modern Astrometry, F. V. Prochazka and R. H. Tucker, eds., pp. 197-217, 1978.

8. M. Gai, S. Casertano, D. Carollo, and M. G. Lattanzi, "Location Estimators for Interferometric Fringes," Publ. Astron. Soc. Pac. 110, pp. 848-862, July 1998.

9. R. A. Mendez, J. F. Silva, and R. Lobos, "Analysis and Interpretation of the Cramér-Rao Lower-Bound in Astrometry: One-Dimensional Case," Publ. Astron. Soc. Pac. 125, pp. 580-594, May 2013.

10. C. M. Will, "The confrontation between general relativity and experiment," Living Reviews in Relativity $\mathbf{9}(3), 2006$.

11. M. Gai, A. Vecchiato, A. Riva, F. Lattanzi, M. G. Landini, B. Bucciarelli, D. Busonero, M. Crosta, S. Liao, G. Luo, H. Mana, M. Pisani, Z. Qi, C. P. Sasso, Z. Tang, and Y. Yu, "Progress on the Astrometric Gravitation Probe design," in This Conference, SPIE Conference Series, 2020.

12. D. Korsch, "Anastigmatic three-mirror telescope.," Appl. Opt. 16, pp. 2074-2077, Aug. 1977.

13. D. Korsch, "Design and optimization technique for three-mirror telescopes," Appl. Opt. 19, pp. 3640-3645, Nov. 1980.

14. G. D. Racca, R. Laureijs, L. Stagnaro, et al., "The Euclid mission design," in Space Telescopes and Instrumentation 2016: Optical, Infrared, and Millimeter Wave, Proc. SPIE 9904, p. 99040O, July 2016. 
15. J. W. Contreras and P. A. Lightsey, "Optical design and analysis of the James Webb Space Telescope: optical telescope element," in Proc. SPIE, J. M. Sasian, R. J. Koshel, P. K. Manhart, and R. C. Juergens, eds., Society of Photo-Optical Instrumentation Engineers (SPIE) Conference Series 5524, pp. 30-41, Oct. 2004.

16. P. S. Davila, B. J. Bos, J. Contreras, et al., "The James Webb Space Telescope science instrument suite: an overview of optical designs," in Proc. SPIE, J. C. Mather, ed., Society of Photo-Optical Instrumentation Engineers (SPIE) Conference Series 5487, pp. 611-627, Oct. 2004.

17. M. Gai, A. Vecchiato, S. Ligori, A. Sozzetti, and M. G. Lattanzi, "Gravitation Astrometric Measurement Experiment," Exp. Astron., 2012.

18. M. Gai, A. Vecchiato, A. Riva, M. G. Lattanzi, A. Sozzetti, M. T. Crosta, and D. Busonero, "Astrometric tests of general relativity in the solar system," Journal of Physics: Conference Series 490, p. 012240, mar 2014.

19. A. Riva, M. Gai, F. Landini, et al., "AGP (Astrometric Gravitation Probe) optical design report," in Proc. SPIE, Society of Photo-Optical Instrumentation Engineers (SPIE) Conference Series 9907, p. 990742, Aug. 2016.

20. F. Landini, A. Riva, M. Gai, et al., "Stray light evaluation for the astrometric gravitation probe mission," in Proc. SPIE, Society of Photo-Optical Instrumentation Engineers (SPIE) Conference Series 9907, p. 990741, Aug. 2016.

21. K. P. Thompson, T. Schmid, and J. P. Rolland, "The misalignment induced aberrations of TMA telescopes," Optics Express 16, p. 20345, Nov. 2008.

22. L. Calamai, S. Barsotti, E. Fossati, et al., "Ring-field TMA for PRISMA: theory, optical design, and performance measurements," in Proc. SPIE, Society of Photo-Optical Instrumentation Engineers (SPIE) Conference Series 9626, p. 96261Z, Sept. 2015.

23. R. Grange, B. Milliard, S. Vivès, et al., "Zero distortion three mirror telescope designed for the Dark Universe Explorer (DUNE) space mission," in Proc. SPIE, Society of Photo-Optical Instrumentation Engineers (SPIE) Conference Series 6265, p. 626549, June 2006.

24. R. Content, "Durham optical design of EUCLID, the merged SPACE/DUNE ESA Dark Energy Mission," in Proc. SPIE, Society of Photo-Optical Instrumentation Engineers (SPIE) Conference Series 7010, p. 701025, July 2008.

25. J. Lee and S.-M. Yu, "Analytic design procedure of three-mirror telescope corrected for spherical aberration, coma, astigmatism, and petzval field curvature," Journal of the Optical Society of Korea 13, pp. 184-192, 062009.

26. D. Busonero, M. Gai, D. Gardiol, et al., "Chromaticity in all-reflective telescopes for astrometry," Astron. Astrophys. 449, pp. 827-836, Apr. 2006.

27. M. Gai, D. Busonero, and R. Cancelliere, "Performance of an Algorithm for Estimation of Flux, Background, and Location on One-dimensional Signals," Publ. Astron. Soc. Pac. 129, p. 054502, May 2017. 